\title{
Mudas de Jasminum mesnyi Hance produzidas com substratos à base de lodo de esgoto compostado
}

\begin{abstract}
Maurício B. Scheer ${ }^{1}$, Charles Carneiro1, Otávio A. Bressan ${ }^{2}$ \& Kaline G. dos Santos ${ }^{2}$
RESUMO

Entre as alternativas de disposição de resíduos urbanos está o reaproveitamento do lodo de esgoto no cultivo de plantas ornamentais e florestais. Objetivou-se, com este trabalho, avaliar o crescimento de Jasminum mesnyi (jasmim amarelo) em substratos à base de lodo de esgoto aeróbio compostado com resíduos de podas de árvores trituradas, com diferentes níveis de fertilizante e comparar o desempenho em relação às mudas produzidas em substrato comercial à base de casca de Pinus compostada e

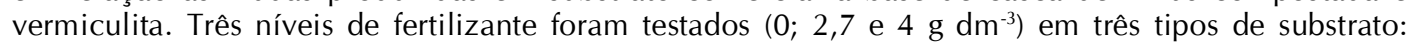
comercial e compostos à base de podas e lodo nas proporções $3: 1(\mathrm{v} / \mathrm{v})$ e $2: 1(\mathrm{v} / \mathrm{v})$. Foram mensuradas as seguintes variáveis: somatória do comprimento dos ramos, altura e biomassa aérea seca (folhas e ramos). O crescimento obtido com o uso dos compostos à base de lodo de esgoto foi superior ao obtido com o substrato comercial para todos os níveis de fertilização. Os maiores crescimentos das mudas de jasmim amarelo foram observados com os compostos nas proporções de 3:1 e 2:1 (v/v) associados à adição de $4 \mathrm{~g} \mathrm{dm}^{-3}$ de fertilizante e com o composto na proporção de 2:1 com a adição de $2,7 \mathrm{~g} \mathrm{dm}^{-3}$ de fertilizante.
\end{abstract}

Palavras-chave: viveiro florestal, biossólido, crescimento

\section{Seedlings of Jasminum mesnyi Hance produced in substrates based on composted sewage sludge}

\begin{abstract}
Use of sewage sludge is one of the alternatives for the disposal of urban waste. Sludge can be used for cultivation of ornamental and forest plants. The aim of this study was to evaluate the use of composted substrates based on sewage sludge and crushed tree pruning for the production of Jasminum mesnyi seedlings (Yellow jasmine) and to compare growth rates with a commercial substrate based on composted Pinus bark and vermiculite. Three levels of fertilization $\left(0 ; 2.7 \mathrm{e} 4 \mathrm{~g} \mathrm{dm}^{-3}\right)$ and three types of substrates were tested: commercial, and 3:1 (v/v) and 2:1 (v/v) composted substrate based on crushed tree pruning and sewage sludge. Sum of branch length, height and dried aerial biomass (leaves and branches) were measured. Growth rates were higher on composted sewage sludge than on commercial substrate for all fertilization levels. Highest growth rates were observed in the $3: 1$ and $2: 1(\mathrm{v} / \mathrm{v})$ treatments plus $4 \mathrm{~g} \mathrm{dm}^{-3} \mathrm{of}^{-1}$ fertilizer as well as in the $2: 1(\mathrm{v} / \mathrm{v})$ treatment plus $2.7 \mathrm{~g} \mathrm{dm}^{-3}$ of fertilizer.
\end{abstract}

Key words: forest nursery, biosolids, growth 


\section{INTRODUÇÃO}

A necessidade de diminuir os impactos negativos das atividades humanas sobre o meio ambiente, cada vez maiores devido ao crescimento populacional tem também, se estendido ao gerenciamento e disposição de resíduos urbanos. Uma das atividades necessárias para diminuir este impacto se refere à coleta e ao tratamento de esgoto. No entanto, este processo resulta em subprodutos sólidos, entre eles o lodo de esgoto (ou biossólido) que são passivos ambientais e devem ser dispostos de forma adequada (Sperling \& Andreoli, 2001).

Considerando as grandes áreas requeridas para sua armazenagem e visando atender às questões ambientais, opções para disposição final do lodo de esgoto se fazem necessárias. Segundo Augusto (2003) estudos a fim de viabilizar sua utilização agrícola representam uma demanda atual, visto que os sistemas de produção são favorecidos pelo uso de insumos regionais e de baixo custo. O uso para fins florestais também deve ser considerado haja vista que os biossólidos podem melhorar a fertilidade de solos florestais (Vaz \& Gonçalves, 2002), geralmente muito intemperizados e originados de materiais quimicamente pobres, que lhes conferem baixa capacidade de troca catiônica e restrita disponibilidade de nutrientes.

O lodo de esgoto é um resíduo orgânico passível de substituir, parcial ou totalmente, os fertilizantes minerais, por ser rico em matéria orgânica e nutrientes, desde que atenda aos requisitos necessários quanto à concentração de metais pesados e patógenos (Silva et al., 2002). Para Morais et al. (1997), Faustino et al. (2005), Cunha et al. (2006), Assenheimer (2009) e Neves et al. (2010) uma das alternativas viáveis para a disposição deste resíduo é seu uso como componente de substratos para o cultivo de mudas, tendo em vista a economia de fertilizantes que este material proporciona (Morais et al., 1997; Bonnet et al., 2002; Silva et al., 2002; Nascimento et al., 2004; Rocha et al., 2004), além dos benefícios ambientais (Guerrini \& Trigueiro, 2004).

A espécie Jasminum mesnyi Hance, conhecida como jasmim amarelo, é um arbusto de textura semi-herbácea pertencente à família Oleaceae, escandente, originário da China, com muitos ramos longos de dois a três metros de comprimento, de folhagem e florescimento decorativos. Possui folhas densas, brilhantes, com três folíolos lisos e verde-escuros, contando ainda com a formação variegada de folhas de cor verde e amarelada. Apresenta rápido crescimento e pode ser utilizado como cercaviva, arbusto informal e até como trepadeira, desde que tenha suporte adequado. Outra opção apontada é seu uso para controle de erosão e embelezamento de barrancos e taludes. Multiplica-se facilmente por estacas cortadas após o florescimento (Lorenzi \& Souza, 2001).

Objetivou-se, com este trabalho, avaliar o crescimento do jasmim amarelo em substratos à base de lodo de esgoto aeróbio compostado com resíduos de podas de árvores trituradas, com diferentes níveis de fertilizante e comparar o desempenho em relação às mudas produzidas em substrato comercial à base de casca de Pinus compostada e vermiculita, amplamente utilizado em viveiros de produção de mudas.

\section{MATERIAL E MÉTODOS}

O experimento foi conduzido em casa de vegetação do Viveiro Florestal do Passaúna, pertencente à Companhia de Saneamento do Paraná - SANEPAR, entre junho e setembro de 2008 e em área de rustificação, entre setembro e novembro de 2008, no município de Araucária, Paraná. O viveiro se localiza nas coordenadas $25^{\circ} 32^{\prime} 03^{\prime \prime} \mathrm{S}$ e $49^{\circ} 23^{\prime} 15^{\prime \prime} \mathrm{O}$, a uma altitude de 900 m. O clima da região, segundo Köppen, é Cfb, com temperatura média anual entre 16 e $18^{\circ} \mathrm{C}$ e precipitação anual média entre 1400 e $1600 \mathrm{~mm}$.

Três tipos de substrato foram testados: (1) substrato comercial de casca de Pinus compostada e vermiculita; (2) substrato à base de resíduos de podas de árvores trituradas compostadas com lodo de esgoto aeróbio da ETE (Estação de Tratamento de Esgoto) Belém/Curitiba/SANEPAR na proporção 3:1 (v:v) e (3) substrato à base de resíduos de poda de árvores trituradas compostadas com lodo de esgoto aeróbio - proporção 2:1 (v:v). Ambos os substratos à base de lodo de esgoto foram peneirados em malha de $2 \mathrm{~mm}$. Cada substrato foi submetido a três níveis de fertilização: (1) sem fertilizante; (2) dose normal, com 2,7 g de fertilizante granulado $\left(\mathrm{N} ; \mathrm{P}_{2} \mathrm{O}_{5} ; \mathrm{K}_{2} \mathrm{O}-15-9-12\right)$ de liberação lenta (5-6 meses) por $\mathrm{dm}^{3} \mathrm{e}$ (3) dose alta, com 4,0 g do mesmo fertilizante por $\mathrm{dm}^{3}$, totalizando nove tratamentos com oito repetições. As análises das concentrações de macro e micronutrientes dos substratos foram realizadas no Laboratório de Nutrição de Plantas - UFPR, segundo Martins \& Reissmann (2007), conforme apresentado nas Tabelas 1 e 2.

Considerando as concentrações totais de metais pesados (análises realizadas no Laboratório de Pesquisas Hidrogeológicas da Universidade Federal do Paraná - UFPR, segundo metodologia da APHA (1998), Tabela 3, e parâmetros parasitológicos (ovos de helmintos) (análises realizadas no Laboratório de Parasitologia Molecular, segundo metodologia

Tabela 1. Concentrações totais de macro e micronutrientes nos substratos testados (03/2008)

\begin{tabular}{|c|c|c|c|c|c|c|c|c|c|}
\hline \multirow{2}{*}{$\begin{array}{c}\text { Tratamentos } \\
\text { Substratos/dose de fertilizante }\left(\mathrm{g} \mathrm{dm}^{-3}\right)\end{array}$} & $\mathbf{N}$ & $\mathbf{P}$ & $\mathbf{K}$ & $\mathrm{Ca}$ & $\mathrm{Mg}$ & $\mathrm{Fe}$ & $\mathrm{Mn}$ & $\mathrm{Cu}$ & $\mathrm{Zn}$ \\
\hline & \multicolumn{3}{|c|}{$\left(g_{k g}^{-1}\right)$} & \multicolumn{6}{|c|}{$\left(\mathrm{mg} \mathrm{kg}^{-1}\right)$} \\
\hline Comercial /0 & 5,8 & 2,6 & 2,1 & 12,9 & 9,23 & 5780 & 78 & 12 & 9 \\
\hline Comercial /2,7 & 5,0 & 2,2 & 2,8 & 11,5 & 9,86 & 7475 & 117 & 18 & 13 \\
\hline Comercial /4,0 & 5,7 & 2,5 & 3,5 & 6,5 & 5,63 & 6266 & 105 & 27 & 16 \\
\hline Composto $3: 1 / 0$ & 19,6 & 8,0 & 2,7 & 17,1 & 4,49 & 10793 & 371 & 66 & 103 \\
\hline Composto $3: 1 / 2,7$ & 20,6 & 8,2 & 4,9 & 19,4 & 5,27 & 9233 & 378 & 72 & 100 \\
\hline Composto $3: 1 / 4,0$ & 20,9 & 8,3 & 6,2 & 18,2 & 4,98 & 8887 & 367 & 86 & 105 \\
\hline Composto $2: 1 / 0$ & 19,3 & 8,4 & 2,9 & 16,0 & 4,17 & 10388 & 395 & 73 & 117 \\
\hline Composto 2:1/2,7 & 20,1 & 8,5 & 4,6 & 16,8 & 4,40 & 9873 & 406 & 86 & 117 \\
\hline Composto $2: 1 / 4,0$ & 22,0 & 8,3 & 5,4 & 16,0 & 4,47 & 10456 & 385 & 38 & 118 \\
\hline
\end{tabular}


Tabela 2. Propriedades químicas do substrato comercial com fertilização mineral comumente utilizado nos viveiros da SANEPAR

\begin{tabular}{|c|c|c|c|c|c|c|c|c|c|c|c|c|c|}
\hline \multirow{2}{*}{ Amostra } & \multicolumn{2}{|c|}{$\mathrm{pH}$} & $\mathrm{Al}^{+3}$ & $\mathrm{H}^{+}+\mathrm{Al}^{+3}$ & $\mathrm{Ca}^{+2}$ & $\mathrm{Mg}^{+2}$ & $\mathrm{~K}^{+}$ & SB & $T$ & \multirow{2}{*}{$\begin{array}{c}P \\
\mathrm{mg} \mathrm{dm}^{-3}\end{array}$} & \multirow{2}{*}{$\underset{g{d m^{-3}}^{C}}{C}$} & & \multirow{2}{*}{$\mathrm{Ca} / \mathrm{Mg}$} \\
\hline & $\mathrm{CaCl}_{2}$ & SMP & \multicolumn{7}{|c|}{$\mathrm{cmol}_{\mathrm{c}} \mathrm{dm}^{-3}$} & & & $\%$ & \\
\hline $\begin{array}{l}\text { Comercial } \\
/ 2,7\left(\mathrm{~g} \mathrm{dm}^{-3}\right)\end{array}$ & 5,9 & 6,2 & 0 & 4,2 & 18,3 & 5,1 & 1,2 & 24,6 & 28,8 & 721,8 & 133,8 & 85 & 3,6 \\
\hline
\end{tabular}

descrita por Soccol et al. (2000), Tabela 4, os substratos higienizados através da compostagem estavam aptos para a utilização agrícola, atendendo às Resoluções do Conselho Nacional de Meio Ambiente n.375/2006 (CONAMA, 2006) e Secretaria Estadual de Meio Ambiente - Paraná (SEMA/PR) n.021/ 2009 (Paraná, 2009). A produção padrão de mudas nos viveiros florestais da SANEPAR utiliza a dose de 2,7 $\mathrm{g}$ do fertilizante granulado citado acima, em conjunto com o substrato comercial de casca de Pinus compostada e vermiculita. A dose de $4 \mathrm{~g}$ (50\% maior) foi escolhida para testar se maiores níveis de fertilizante resultam em maior desenvolvimento das mudas.

Tabela 3. Concentrações totais de metais pesados em amostra composta pelos dois compostos à base de lodo de esgoto e de poda de árvores trituradas, utilizados como substratos. Os números entre parênteses representam os limites estabelecidos pela Resolução CONAMA n. 375/ 2006

\begin{tabular}{ccccc}
\hline $\mathbf{A l}$ & $\mathbf{C d}$ & $\mathbf{P b}$ & $\mathrm{Na}$ & $\mathbf{C r}$ \\
\hline \multicolumn{5}{c}{$\%$} \\
0,86 & $<0,001$ & $<0,005$ & $<0,01$ & $<0,005$ \\
& $(0,0039)$ & $(0,03)$ & & $(0,1)$ \\
\hline
\end{tabular}

Tabela 4. Parâmetros parasitológicos (ovos viáveis por grama de sólidos totais) dos compostos à base de lodo de esgoto e de poda de árvores trituradas utilizados como substratos

\begin{tabular}{lcc}
\multirow{2}{*}{ Helminto } & \multicolumn{2}{c}{ Composto } \\
\cline { 2 - 3 } & $\mathbf{3 : 1}$ & $\mathbf{2 : 1}$ \\
Ascaris sp. & 0 & 0,05 \\
Toxocara sp. & 0 & 0,01 \\
Trichuris trichiura & 0 & 0,00 \\
Trichuris vulpis & 0 & 0,01 \\
Trichuroidea & 0 & 0,01 \\
Hymenolepis diminuta & 0 & 0,01 \\
Taenia sp. & 0 & 0,00 \\
\hline Total geral & 0 & 0,10 \\
\hline Protozoários & 0,28 & 0,38 \\
\hline
\end{tabular}

Observação: análises realizadas em triplicata

Limite estabelecido pela Resolução CONAMA n ${ }^{0} 375 / 2006$ : ovos viáveis de helmintos $<0,25$ ovo por grama de sólidos totais
As estacas de Jasminum mesnyi foram produzidas através de ramos retirados de indivíduos oriundos de plantios da Prefeitura Municipal de Curitiba. As estacas com dimensões de aproximadamente $17 \times 0,8 \mathrm{~cm}$ foram confeccionadas pela manhã e transportadas em recipiente úmido até o viveiro, sendo então podadas as folhas e plantadas no período da tarde. $\mathrm{O}$ plantio foi realizado em tubetes plásticos com formato cônico e capacidade de $110 \mathrm{~cm}^{3}$, encaixados em bandejas, inserindo-se cerca de metade da estaca no substrato. A irrigação foi realizada duas vezes por dia através de microaspersores, tanto na casa de vegetação quanto na área de rustificação, sendo uma no início da manhã e outra no final da tarde.

Para a avaliação foram mensuradas pelo menos nove plantas úteis de cada repetição (oito repetições por tratamento), descartando-se as plantas das bordaduras. Ao atingirem cinco meses de idade as mudas foram mensuradas para obtenção das seguintes variáveis: altura, somatória do comprimento dos ramos e biomassa seca aérea (folhas e ramos pertencentes às novas brotações). Com vista à obtenção dos dados de biomassa, foram cortadas todas as novas brotações das plantas de cada uma das repetições, deixando-se apenas a estaca inicial e, em seguida, dispostas em embalagens de papel submetidas à secagem em estufa a $60^{\circ} \mathrm{C}$, por $72 \mathrm{~h}$. O experimento foi conduzido em delineamento inteiramente casualizado em arranjo fatorial $3 \times 3$, com três tipos de substrato e três níveis de fertilização. Após a verificação da homogeneidade das variâncias e outras condicionantes através do teste de Bartlett, foram realizados análises de variância e testes de Tukey (5\% de probabilidade) para comparação das médias. Para todas as variáveis, nas quais se detectaram interações entre os fatores, foram comparados os níveis de um fator dentro do outro. Regressões polinomiais foram realizadas para avaliar os efeitos dos níveis de fertilizante (fator quantitativo) sobre as variáveis.

\section{RESULTADOS E DISCUSSÃO}

Para a variável somatória do comprimento dos ramos, o substrato comercial respondeu a todos os níveis de fertilização testados, obtendo-se então maiores valores com o aumento da dose de fertilizante (Tabela 5 e Figura 1A). O mesmo padrão foi

Tabela 5. Médias de altura e somatória do comprimento dos ramos de mudas de Jasminum mesnyi, cultivadas em três tipos de substrato e enriquecidas com diferentes níveis de fertilizante mineral

\begin{tabular}{|c|c|c|c|c|c|c|}
\hline \multirow{3}{*}{ Substrato } & \multicolumn{3}{|c|}{ Somatória do comprimento dos ramos (cm) } & \multicolumn{3}{|c|}{ Altura (cm) } \\
\hline & \multicolumn{6}{|c|}{ Fertilizante $\left(\mathrm{g} \mathrm{dm}^{-3}\right)$} \\
\hline & 0 & 2,7 & 4 & $\mathbf{0}$ & 2,7 & 4 \\
\hline Comercial & $21,5 \pm 1,7 \mathrm{cC}$ & $57,1 \pm 1,6 \mathrm{cB}$ & $73,1 \pm 2,5 \mathrm{bA}$ & $17,1 \pm 0,6 \mathrm{cB}$ & $36,4 \pm 0,6 \mathrm{cA}$ & $37,2 \pm 0,8 \mathrm{bA}$ \\
\hline Composto $3: 1$ & $60,1 \pm 2,5 b C$ & $82,3 \pm 3,1 \mathrm{bB}$ & $97,0 \pm 3,2 \mathrm{aA}$ & $33,1 \pm 0,4 b C$ & $39,1 \pm 1,6 b B$ & $46,8 \pm 0,9 \mathrm{aA}$ \\
\hline Composto $2: 1$ & $88,3 \pm 3,2 \mathrm{aB}$ & $93,0 \pm 2,3 \mathrm{aAB}$ & $96,0 \pm 4,1 \mathrm{aA}$ & $43,5 \pm 1,3 \mathrm{aB}$ & $47,6 \pm 1,1 \mathrm{aA}$ & $45,0 \pm 1,6 \mathrm{aAB}$ \\
\hline
\end{tabular}

As médias seguidas da mesma letra maiúscula na linha e da mesma letra minúscula na coluna não diferem entre si, pelo teste de Tukey a 0,05 de probabilidade 
A.

Comercial

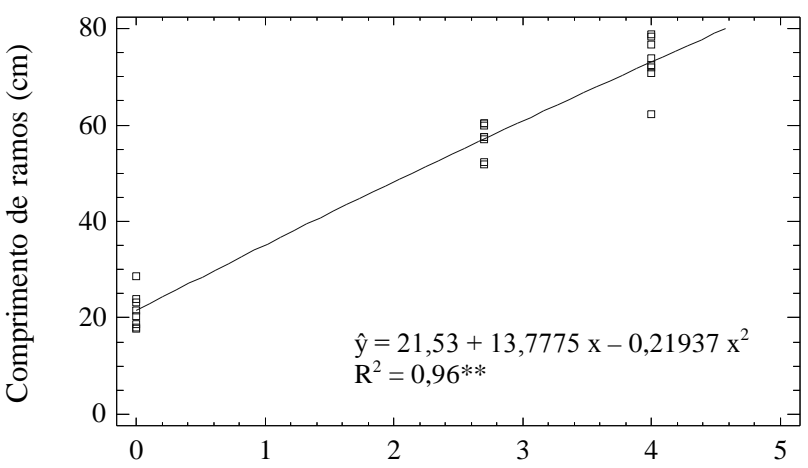

B.

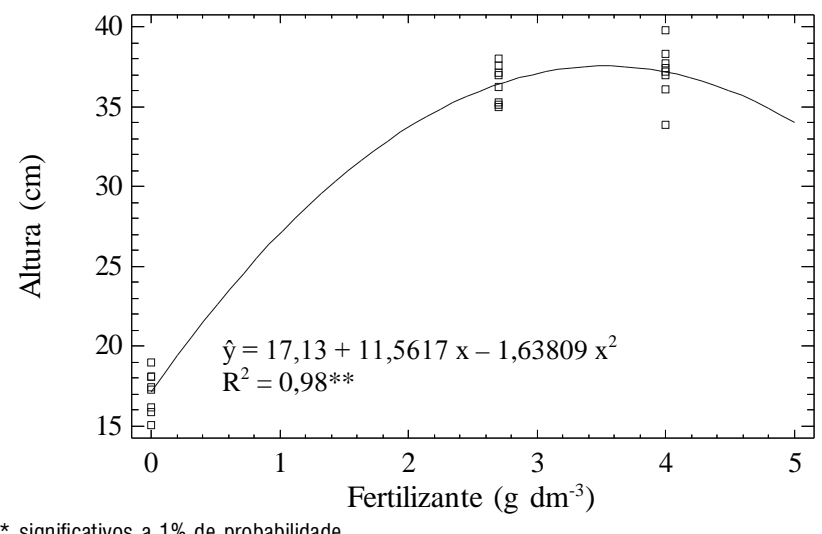

Composto 3:1
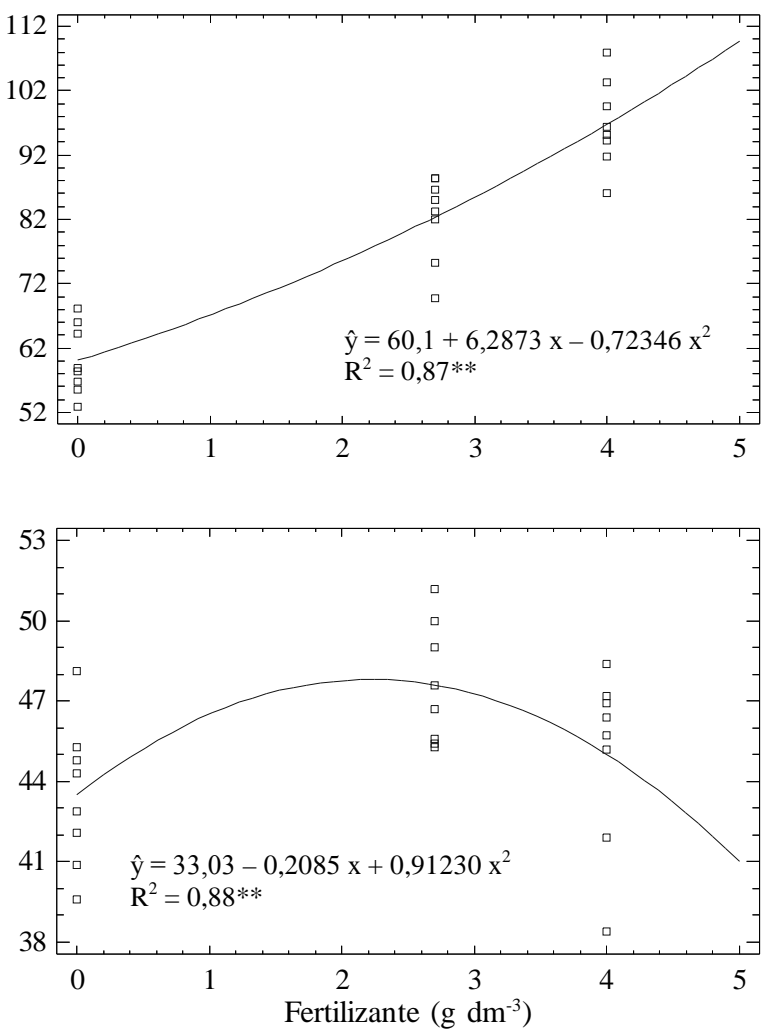

Figura 1. Regressões polinomiais significativas para somatória de comprimento dos ramos (A) e alturas (B) das mudas de Jasminum mesnyi cultivadas nos substratos enriquecidos com diferentes níveis de fertilizante mineral

observado para o composto à base de lodo na proporção $3: 1$, porém ambos os compostos com lodo (3:1 e 2:1) apresentaram maiores comprimentos dos ramos em relação ao substrato comercial (Tabela 5 e Figura 1A). No entanto, não foi obtido um $\mathrm{R}^{2}$ significativo na análise de regressão polinomial com os níveis de fertilizante testados no composto $2: 1(\hat{y}=88,26+$ $1,4889 x+0,10354 x^{2} ; R^{2}=0,18$ n.s.).

Ao se comparar os três substratos sem adição de fertilizante, fica evidente a superioridade dos compostos, visto que os mesmos proporcionaram crescimento até $311 \%$ superior ao substrato comercial, como observado no composto 2:1. Quando o lodo é aplicado no solo como única fonte de $\mathrm{N}$ para as plantas, as quantidades de micronutrientes adicionadas por este insumo são, na maioria das vezes, suficientes para atender às demandas nutricionais das plantas (Andreoli et al., 2001).

O somatório do comprimento dos ramos obtido com o composto 3:1 com adição de $4 \mathrm{~g} \mathrm{dm}^{-3}$ de fertilizante, foi superior até mesmo ao composto $2: 1$, porém não diferiu deste estatisticamente pelo teste de Tukey, sendo $32 \%$ maior ao obtido ao mesmo nível de fertilização com o substrato comercial. Os valores para esta variável obtidos com o composto na proporção $2: 1 \operatorname{com} 2,7 \mathrm{~g} \mathrm{dm}^{-3}$ de fertilizante foram $63 \%$ superiores ao substrato comercial com o mesmo nível de fertilização, considerado produção padrão dos viveiros da SANEPAR (Tabela 5). Isto se deve, provavelmente, às maiores concentrações de macronutrientes, principalmente $\mathrm{N}$ e $\mathrm{P}$
(Andreoli et al., 2001) e de micronutrientes (Fe, Mn, Cu e Zn) presentes no lodo de esgoto (Tabela 1).

Os tratamentos que proporcionaram as maiores alturas foram obtidos também com o uso de compostos à base de lodo de esgoto, sendo superiores ao substrato comercial para todos os níveis de fertilização, ficando a superioridade ainda mais evidente ao se analisar os resultados para os substratos sem a adição de fertilizante (Tabela 5 e Figura 1B). A maior média observada foi obtida com o composto na proporção 2:1 com 2,7 $\mathrm{g} \mathrm{dm}^{-3}$ de fertilizante (Tabela 5), denotando que a dose de fertilizante superior a esta, configura apenas um consumo de luxo, podendo provocar imobilização de nutrientes ou até certa toxicidade (Scheer et al., 2010), uma vez que o valor observado não apresentou diferenças significativas (Tabela 5). No entanto, para a variável altura o valor de $\mathrm{R}^{2}$ obtido foi baixo para a regressão polinomial com os níveis de fertilizante testados no composto $2: 1\left(\hat{y}=43,5+3,8870 x-0,87722 x^{2} ; R^{2}=0,30 ; P<\right.$ $0,05)$.

Com o aumento das doses de fertilizante ocorreu tendência de incremento de biomassa foliar, sobretudo para o substrato 3:1 (Tabela 6 e Figura 2A). Os valores mais elevados foram obtidos com os compostos à base de lodo, independente do nível de fertilização. Sem a adição de fertilizante o valor observado para o composto 2:1 foi cinco vezes maior ao obtido com o substrato comercial, demonstrando a superioridade nutricional dos compostos (Tabela 6). Considerando que a utilização do substrato comercial é recomendada pelo fabricante 
Tabela 6. Médias de biomassa seca de folhas e ramos de mudas de Jasminum mesnyi, cultivadas em três tipos de substrato e enriquecidas com diferentes níveis de fertilizante mineral

\begin{tabular}{|c|c|c|c|c|c|c|}
\hline \multirow{3}{*}{ Substrato } & \multicolumn{3}{|c|}{ Folhas (g) } & \multicolumn{3}{|c|}{ Ramos (g) } \\
\hline & \multicolumn{6}{|c|}{ Fertilizante $\left(\mathrm{g} \mathrm{dm}^{-3}\right)$} \\
\hline & 0 & 2,7 & 4 & 0 & 2,7 & 4 \\
\hline Comercial & $2,1 \pm 0,1 \mathrm{cB}$ & $6,5 \pm 0,1 \mathrm{cA}$ & $7,4 \pm 0,1 \mathrm{bA}$ & $0,7 \pm 0,2 c B$ & $4,0 \pm 0,1 \mathrm{cA}$ & $4,9 \pm 0,1 \mathrm{bA}$ \\
\hline Composto 3:1 & $5,9 \pm 0,1 b C$ & $9,4 \pm 0,1 b B$ & $12,8 \pm 0,1 \mathrm{aA}$ & $3,0 \pm 0,1 \mathrm{bC}$ & $5,6 \pm 0,1 \mathrm{bB}$ & $8,7 \pm 0,1 \mathrm{aA}$ \\
\hline Composto 2:1 & $10,6 \pm 0,1 \mathrm{aB}$ & $12,2 \pm 0,1 \mathrm{aA}$ & $12,9 \pm 0,1 \mathrm{aA}$ & $5,7 \pm 0,1 \mathrm{aB}$ & $7,3 \pm 0,1 \mathrm{aA}$ & $8,1 \pm 0,1 \mathrm{aA}$ \\
\hline
\end{tabular}

As médias seguidas da mesma letra maiúscula na linha e da mesma letra minúscula na coluna não diferem entre si, pelo teste de Tukey a 0,05 de probabilidade

com a adição de fertilizante mineral e se comparando o composto 2:1 com 2,7 $\mathrm{g} \mathrm{dm}^{-3}$ de fertilizante com o substrato comercial com o mesmo nível de fertilização, nota-se que os resultados com o composto com lodo foram $88 \%$ superiores (Tabela 6). Este resultado foi estatisticamente igual aos obtidos nos compostos 2:1 e 3:1 enriquecidos com $4 \mathrm{~g} \mathrm{dm}^{-3} \mathrm{de}$ fertilizante, indicando ser a dose mais adequada entre as testadas, proporcionando bom crescimento das mudas, com economia de fertilizante mineral.

A biomassa dos ramos respondeu de maneira semelhante à observada para a biomassa das folhas (Figura 2B), embora o valor de $\mathrm{R}^{2}(0,35 ; \mathrm{p}<0,05)$ tenha sido baixo para a regressão com o substrato comercial $\left(\hat{y}=0,199+0,1256 x-0,01002 x^{2}\right)$. Neste caso, as maiores médias também foram obtidas com o uso de compostos à base de lodo. $\mathrm{O}$ composto na proporção 2:1 enriquecido com $2,7 \mathrm{~g} \mathrm{dm}^{-3}$ provocou, em média, $83 \%$ de

A.

\section{Comercial}

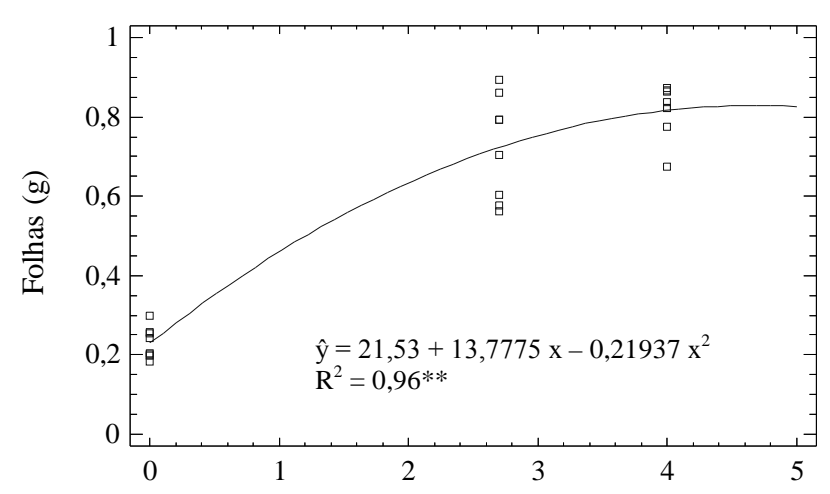

B.

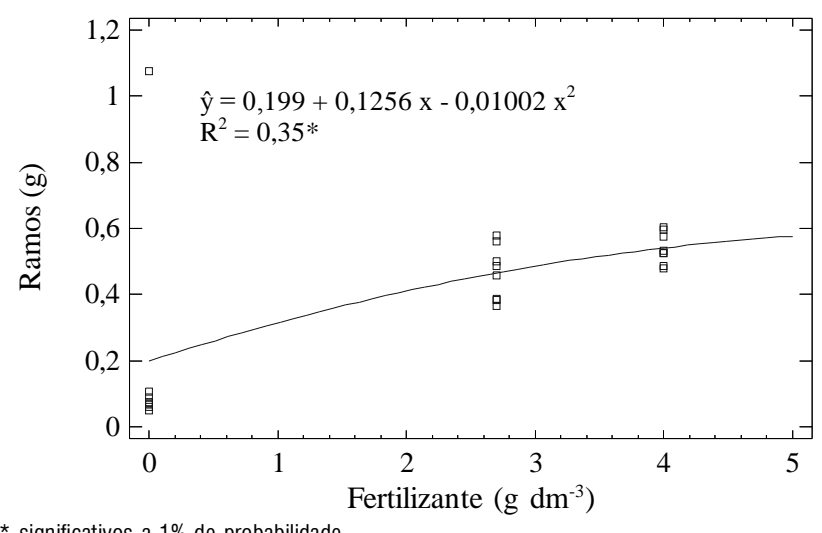

A. superioridade em relação ao substrato comercial com o mesmo nível de fertilização (Tabela 6).

Apesar de significativos, para as variáveis biomassa seca de folhas e de ramos, os valores de $\mathrm{R}^{2}$ foram baixos para regressões com os níveis de fertilizante testados no composto $2: 1\left(\hat{y}=1,183+0,0136 \mathrm{x}+0,01558 \mathrm{x}^{2} ; \mathrm{R}^{2}=0,40, \mathrm{P}<0,01 \mathrm{e} \mathrm{w}=\right.$ $0,635+0,0645 \mathrm{x}+0,00058 \mathrm{x}^{2} ; \mathrm{R}^{2}=0,40 ; \mathrm{P}<0,01 ;$ respectivamente).

Os compostos à base de lodo apresentaram maior biomassa aérea total (folhas + ramos) quando comparados ao substrato comercial, com ou sem fertilização, sendo a diferença das médias até seis vezes maior nos compostos em relação ao substrato comercial sem a adição de fertilizante (Tabela 7). Observou-se tendência a maiores valores para os três substratos testados, à medida em que a dose de fertilizante foi elevada (Figura 3 ), embora o valor de $\mathrm{R}^{2}(0,47 ; \mathrm{p}<0,01)$ tenha sido baixo para a
Figura 2. Regressões polinomiais significativas para biomassa seca de folhas (A) e ramos (B) das mudas de Jasminum mesnyi cultivadas nos substratos enriquecidos com diferentes níveis de fertilizante mineral

\section{Composto $3: 1$}
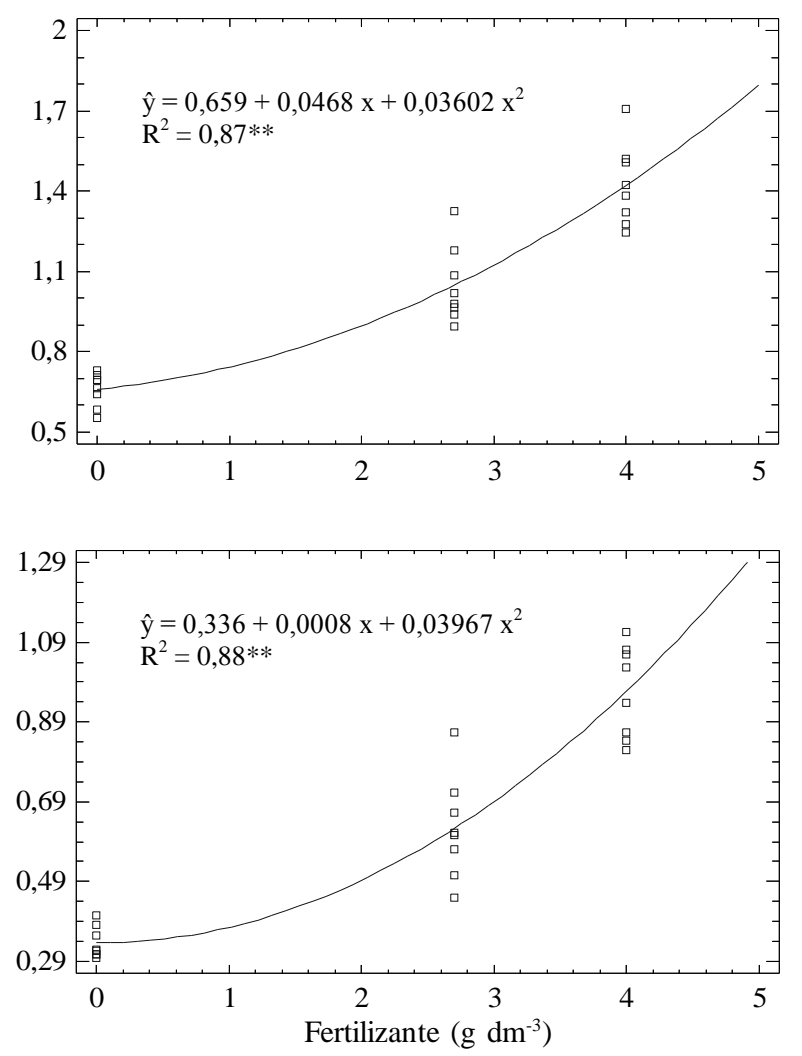
regressão com ocomposto $2: 1\left(\hat{y}=1,821+0,0772 x+0,01631 x^{2}\right)$. O composto 3:1 apresentou grandes diferenças médias de um nível para o outro, obtendo-se, daí, resposta positiva maior ao acréscimo de fertilizante, fato que não ficou tão evidenciado para o composto 2:1 e substrato comercial, pois não se observaram diferenças significativas entre as doses 2,7 e 4 $\mathrm{g} \mathrm{dm}^{-3}$. Com a maior dose de fertilizante mineral o composto $3: 1$ apresentou o maior resultado; entretanto, semelhante estatisticamente aos obtidos pelo composto na proporção 2:1 com as doses de $2,7 \mathrm{e} 4 \mathrm{~g} \mathrm{dm}^{-3}$ (Tabela 7).

Os compostos à base de lodo de esgoto e restos de podas de árvores trituradas, promoveram maior crescimento às mudas

Tabela 7. Médias de biomassa seca aérea total de mudas de Jasminum mesnyi, cultivadas em três tipos de substrato e enriquecidas com diferentes níveis de fertilizante mineral; $\mathrm{n}=8$

\begin{tabular}{lrcc}
\hline \multirow{3}{*}{ Substrato } & \multicolumn{3}{c}{ Biomassa aérea (folhas +ramos) - $\mathbf{g}$} \\
\cline { 2 - 4 } & \multicolumn{3}{c}{ Fertilizante $\left(\mathbf{g ~ d m}^{-\mathbf{3}}\right)$} \\
\cline { 2 - 4 } Comercial & $\mathbf{0}$ & $\mathbf{2 , 7}$ & $\mathbf{4}$ \\
Composto $3: 1$ & $8,8 \pm 0,2 \mathrm{cB}$ & $10,5 \pm 0,1 \mathrm{cA}$ & $12,3 \pm 0,1 \mathrm{bA}$ \\
Composto $2: 1$ & $16,3 \pm 0,1 \mathrm{bB}$ & $15,1 \pm 0,1 \mathrm{bB}$ & $21,5 \pm 0,1 \mathrm{aA}$ \\
\hline
\end{tabular}

As médias seguidas da mesma letra maiúscula na linha e da mesma letra minúscula na coluna não diferem entre si, pelo teste de Tukey a 0,05 de probabilidade
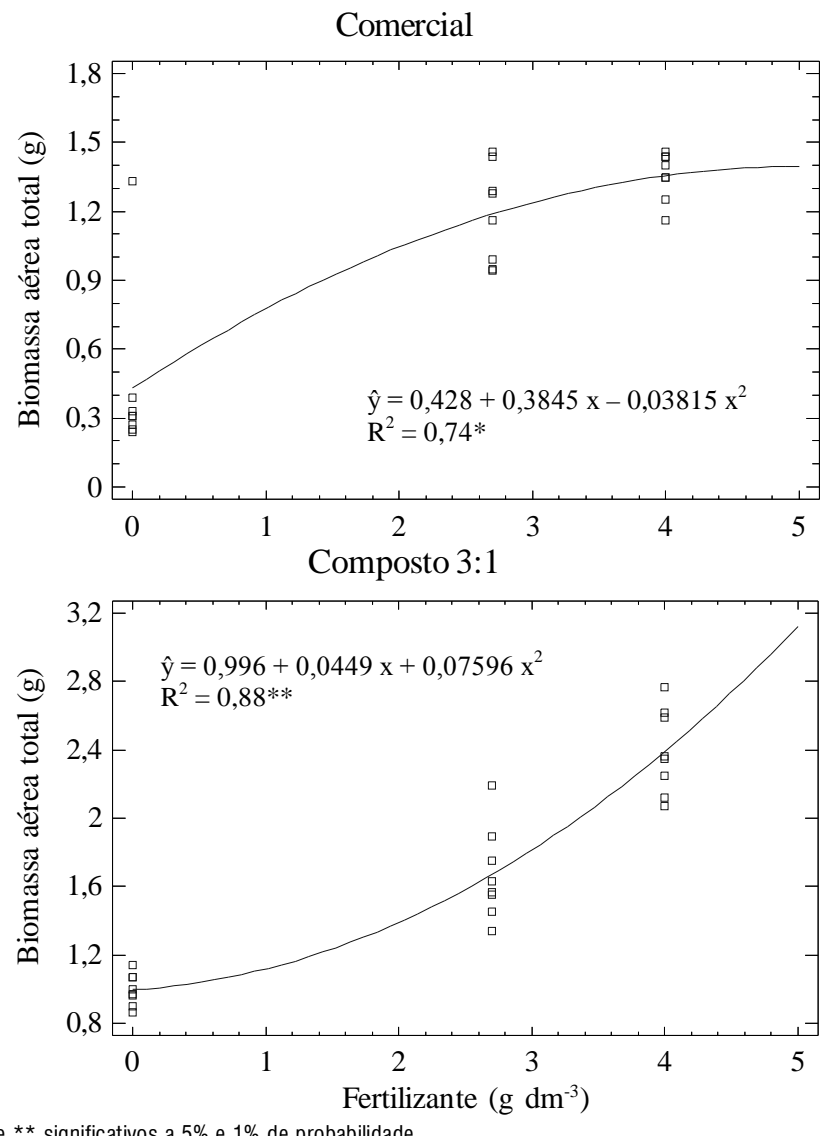

*

Figura 3. Regressões polinomiais significativas para biomassa seca aérea total das mudas de Jasminum mesnyi cultivadas nos substratos enriquecidos com diferentes níveis de fertilizante mineral em razão, provavelmente, das maiores concentrações de nutrientes, oriundas do lodo em relação ao substrato comercial (Tabela 1). Segundo Wilson et al. (2006), compostos à base de lodo e poda de árvores apresentam teores elevados de N, P, K, $\mathrm{Zn}, \mathrm{Cu}, \mathrm{Mn}, \mathrm{Al}$ e Fe. Os macronutrientes N, P e K estão diretamente ligados ao desenvolvimento da planta, uma vez que o nitrogênio é um dos elementos minerais mais requeridos pelas plantas, fazendo parte de proteínas, ácidos nucléicos e muitos outros constituintes vegetais, incluindo membranas e diversos hormônios, além de que sua deficiência provoca redução do crescimento (Souza \& Fernandes, 2006). O fósforo participa de vários processos metabólicos das plantas, como a transferência de energia, síntese de ácidos nucléicos, glicose, respiração, síntese de proteínas, metabolismo de carboidratos e fixação de $\mathrm{N}_{2}$ (Araújo, 2006). Por sua vez, o potássio regula a perda de água atuando na abertura e fechamento dos estômatos e ainda participa da síntese de proteínas, carboidratos e relações osmóticas (Malavolta, 2006).

Fica evidenciada a superioridade nutricional dos compostos à base de lodo de esgoto, uma vez que os resultados obtidos com seu uso foram superiores ao substrato comercial à base de casca de Pinus compostada e vermiculita, para todos os níveis de fertilização (Tabelas 5, 6 e 7). Os melhores resultados foram conseguidos com a utilização dos compostos nas proporções 3:1 e 2:1 com a adição de $4 \mathrm{~g} \mathrm{dm}^{-3} \mathrm{e}$ 2:1 com a adição de $2,7 \mathrm{~g} \mathrm{dm}^{-}$ ${ }^{3}$ de fertilizante, todos produzindo mudas vigorosas e de boa qualidade, sendo recomendado o último tratamento pela economia de fertilizante. Ambos os compostos com lodo sem fertilizante mostraram resultados satisfatórios, sendo que as mudas cultivadas no composto com proporção 3:1 apresentaram resultados superiores ao substrato comercial com a dose de 2,7 $\mathrm{g} \mathrm{dm}^{-3} \mathrm{de}$ fertilizante para a maioria das variáveis.

Os resultados obtidos com o uso de lodo de esgoto neste trabalho, concordam com os obtidos por Wilson et al. (2006) na produção de mudas de Forestiera segregata var. pinerotum, Myrcianthes fragrans e Viburnum obovata. Nóbrega et al. (2007) na produção de mudas de Schinus terebinthifolius. Scheer et al. (2010) na produção de mudas de Paraptadenia rigida e Scheer et al. (2012) na produção de mudas de Lafoensia pacari, usando compostos à base de lodo de esgoto como substratos. Tais resultados corroboram para o uso de biossólidos como componente de substrato para a produção de mudas, pois se trata de uma alternativa economicamente viável aos substratos comerciais (Ingelmo et al., 1998), promovendo economia de insumos e mudas de boa qualidade (Scheer et al., 2011).

\section{CONCLUSÕES}

1. O crescimento das mudas produzidas com o composto à base de lodo de esgoto e restos de podas de árvores trituradas foi consideravelmente maior ao obtido com o uso do substrato comercial.

2. O maior crescimento das mudas de jasmim amarelo foi observado com os compostos nas proporções 3:1 e 2:1 associados à adição de $4 \mathrm{~g} \mathrm{dm}^{-3}$ de fertilizante e com o composto na proporção 2:1 com a adição de $2,7 \mathrm{~g} \mathrm{dm}^{-3}$ de fertilizante. 


\section{LITERATURA CITADA}

Andreoli, C. V.; Pegorini, E. S.; Fernandes, F. Disposição de lodo no solo. In: Andreoli, C. V.; Sperling, M. von; Fernandes, F. (ed.). Lodos de esgotos: Tratamento e disposição final. v.6, Belo Horizonte: UFMG, Companhia de Saneamento do Paraná(SANEPAR), 2001. p.319-395.

APHA - American Public Health Association. Standard methods for the examination of water and wastewater. 20.ed. Washington: APHA, AWWA, WEF, 1998. 1325p.

Araújo, A. P.; Machado, C. T. T. Fósforo. In: Fernandes, M. S. (ed.). Nutrição mineral de plantas. Viçosa: SBCS, 2006. Cap.10, p. 253-280.

Assenheimer, A. Benefícios do uso de biossólidos como substratos na produção de mudas de espécies florestais. Revista Ambiência, v.5, p.321-330, 2009.

Augusto, D. C. C. Utilização de esgotos domésticos tratados através de um sistema biológico na produção de mudas de Croton floribundus Spreng (capixingui) e Copaifera langsdorffii Desf. (copaíba). Revista Árvore, v.27, p.335342, 2003.

Bonnet, B. R. P.; Wisniewski, C.; Reissmann, C. B.; Nogueira, A. C.; Andreoli, C. V.; Barbieri, S. J. Effects of substracts composed of biosolids on the production of Eucalyptus viminalis, Schinus terebinthifolius and Mimosa scabrella seedlings and on the nutritional status of Schinus terebinthifolius seedlings. Water Science and Technology, v.46, p.239-246, 2002.

CONAMA - Conselho Nacional do Meio Ambiente. Resolução n: 375/2006, de 29/8/2006. http://www.mma.gov.br/port/ conama/res/res06/res37506.pdf. 17 Abr. 2012.

Cunha, A. M.; Cunha, G. M.; Sarmento, R. A.; Cunha, G. M.; Amaral, J. F. T. Efeito de diferentes substratos sobre o desenvolvimento de mudas de Acacia sp. Revista Árvore, v.30, p.207-214, 2006.

Faustino, R.; Kato, M. T.; Florencio, L.; Gavazza, S. Lodo de esgoto como substrato para produção de mudas de Senna siamea Lam. Revista Brasileira de Engenharia Agrícola e Ambiental, v.9, p.278-282, 2005.

Guerrini, I. A.; Trigueiro, R. M. Atributos físicos e químicos de substratos compostos por biossólido e casca de arroz carbonizada. Revista Brasileira de Ciência do Solo, v.28, p.1069-076, 2004.

Ingelmo, F.; Canet, R.; Ibanez, M. A.; Pomares, F.; Garcia, J. Use of MSW compost, dried sewage sludge and other wastes as partial substitutes for peat and soil. Bioresource Technology, v.63, p.123-129, 1998.

Lorenzi, H.; Souza, H. M. Plantas ornamentais no Brasil Arbustivas, herbáceas e trepadeiras. 4.ed. Nova Odessa: Instituto Plantarum. 2001, 1120p.

Malavolta, E. Manual de nutrição mineral de plantas. São Paulo: Agronômica Ceres, 2006. 638p.

Martins, A. P. L.; Reissmann, C. B. Material vegetal e as rotinas laboratoriais nos procedimentos químico-analíticos. Scientia Agraria, v.8, p.1-17, 2007.

Morais, S. M. J. de; Ataides, P. R. V.; Garcia, D. C.; Kurtz, F. C.; Oliveira, O. S.; Watzlawick, L. F. Uso do lodo de esgoto da Corsan - Santa Maria (RS), comparada com outros substratos orgânicos. Sanare, v.6, p.44-49, 1997.
Nascimento, C. W. A.; Barros, D. A. S.; Melo, E. E. C.; Oliveira, A. B. Alterações químicas em solos e crescimento de milho e feijoeiro após a aplicação de lodo de esgoto. Revista Brasileira de Ciência do Solo, v.28, p.385-392, 2004.

Neves, J. M. G.; Silva, H. P.; Duarte, R. F. Uso de substratos alternativos para produção de mudas de moringas. Revista Verde de Agroecologia e Desenvolvimento Sustentável, v.5, p.173-177, 2010.

Nóbrega, R. S. A.; Boas, R. C. V.; Nobrega, J. C. A.; Paula, A. M.; Moreira, F. M. S. Utilização de biossólido no crescimento inicial de mudas de aroeira (Schinus terebinthifolius Raddi). Revista Árvore, v.31, p.239-246, 2007.

Paraná. Secretaria de Estado de Meio Ambiente e Recursos Hídricos. Resolução SEMA 021/09. Dispõe sobre licenciamento ambiental, estabelece condições e padrões ambientais e dá outras providências, para empreendimentos de saneamento. Diário Oficial do Estado do Paraná, Curitiba, n.7962, 2009. p.13-16.

Rocha, G. N.; Gonçalves, J. L. M.; Moura, I. M. Mudanças da fertilidade do solo e crescimento de um povoamento de Eucalyptus grandis fertilizado com biossólido. Revista Brasileira de Ciência do Solo, v.28, p.623-639, 2004.

Scheer, M. B.; Carneiro, C.; Bressan, O. A.; Santos, K. G. Crescimento e nutrição de mudas de Lafoensia pacari com lodo de esgoto. Floresta \& Ambiente, v.19, p.55-65, 2012.

Scheer, M. B.; Carneiro, C.; Santos, K. G. Substratos à base de lodo de esgoto compostado na produção de mudas de Paraptadenia rigida (Benth.) Brenan. Scientia Forestalis, v.38, p.637-644, 2010.

Scheer, M. B.; Carneiro, C.; Santos, K. G.; Bressan, O. A.; Horokoski, G. Produção de mudas de Calophyllum brasiliense Cambess com substratos comercial e à base de lodo de esgoto compostado. Revista DAE, v.187, p.55-61, 2011.

Silva, J. E.; Resck, D. V. S.; Sharma, R. D. Alternativa agronômica para o biossólido produzido no Distrito Federal. I. Efeito na produção de milho e na adição de metais pesados em Latossolo no Cerrado. Revista Brasileira de Ciência do Solo, v.26, p.487-495, 2002.

Soccol V. T.; Paulino R. C.; Castro, E. A. Metodologia para análise parasitológica em lodo de esgoto. In: Andreoli, C. V.; Bonnet, B. R. P. (ed.). Manual de métodos para análises microbiológicas e parasitológicas em reciclagem agrícola de lodo de esgoto. 2.ed. Curitiba: SANEPAR, 2000. p.27-41.

Souza, S. R.; Fernandes, M. S. Nitrogênio. In: Fernandes, M. S. (ed.) Nutrição mineral de plantas. Viçosa: SBCS, 2006. Cap. 9, p.215-254.

Sperling, M. von; Andreoli, C. V. Introdução. In: Andreoli, C. V.; Sperling, M. von; Fernandes, F. (ed.). Lodos de esgotos: Tratamento e disposição final. Belo Horizonte: UFMG, SANEPAR, 2001. v.6, p.13-16.

Vaz, L. M. S.; Gonçalvez, J. L. M. Uso de biossólidos em povoamento de eucalipto: Efeito em atributos químicos do solo, no crescimento e na absorção de nutrientes. Revista Brasileira de Ciência do Solo, v.26, p.747-758, 2002.

Wilson, S. B.; Mecca, L. K.; Danielson, H. E.; Graetz, D. A.; Stoffela, P. J. Container and field evaluation of three native shrubs grown in compost-based media. Compost Science \& Utilization, v.14, p.178-183, 2006. 